\title{
A Spectral Survey of Molecules in the Orion Nebula from 455 - 507 GHz: An Inventory of Prebiotic Chemistry
}

Glenn J. White

Physics Department, University of Kent, CT2 7NR England

M. Araki

Institute for Physical Chemistry, University of Basel, Switzerland

J. S. Greaves

Royal Observatory, Blackford Hill, Edinburgh EH9 3HJ, Scotland

M. Ohishi

National Astronomical Observatory of Japan, Mitaka, Japan

\begin{abstract}
The results of a submillimetre wavelength spectral line survey between $455.1-507.4 \mathrm{GHz}$ of the Orion-KL hot cloud core are reported. 254 lines were detected, and are associated with 30 different molecular species or their isotopomeric variants. Apart from the abundant diatomic rotors such as $\mathrm{CO}$ and CS, the spectrum is dominated by $\mathrm{SO}, \mathrm{SO}_{2}$ and $\mathrm{CH}_{3} \mathrm{OH}$ and large organic molecules such as $\left(\mathrm{CH}_{3}\right)_{2} \mathrm{O}$, $\mathrm{CH}_{3} \mathrm{CN}, \mathrm{C}_{2} \mathrm{H}_{3} \mathrm{CN}, \mathrm{C}_{2} \mathrm{H}_{5} \mathrm{CN}$ and $\mathrm{HCOOCH}_{3}$ which make up $\sim 72 \%$ of the total number of lines; unidentified lines $\sim 13 \%$; and other lines the remaining $\sim 15 \%$ of the total. Rotational temperatures and column densities derived using standard rotation diagram analysis techniques were found to range from $70-600 \mathrm{~K}$, and $10^{14}-10^{17} \mathrm{~cm}^{2}$ respectively.
\end{abstract}

\section{Introduction}

Life is fundamentally an accumulation of organic chemical processes of sufficient complexity to be mutating and self-replicating. Although little is known about prebiotic chemistry, the building blocks of cells were probably simple organic compounds created from simpler compounds abundant in space - e.g. mixtures of methane, ammonia, and water exposed to electric discharge or other energy sources are known to yield the amino acids of proteins and the bases of nucleic acids. However, there is a gap in our knowledge of the chemical steps occur between the formation of simple organic compounds and the rise of complexity that could breed life. To date, more than 20 molecules have already been discovered in comets - whose icy composition is analogous to that of interstellar material and indicates that comets are relics of protosolar chemistry. Complex organics such as $\mathrm{HCOOH}$ or $\mathrm{HCOOCH}_{3}$ have been observed, but the chemical inventory is far from complete. In the absence of a ready source of pristine proto-nebular material, molecular line surveys from ground and space-based telescopes pro- 
vide our best, and unbiased view of the molecular constituents of the gas in star forming regions. They sample the chemical inventory of material that must have existed in the early solar system, and help to characterise the reaction pathways that may lead to the formation of complex organic molecules and the interstellar precursors to amino acids.

Table 1. JCMT Orion spectral line survey

\begin{tabular}{|c|c|c|c|c|c|}
\hline Molecule & $\begin{array}{l}\mathrm{N}_{c o l} \\
\mathrm{~cm}^{-3}\end{array}$ & $\begin{array}{l}\text { Error } \\
\mathrm{cm}^{-3}\end{array}$ & $\begin{array}{l}\mathrm{T}_{\text {rot }} \\
\mathrm{K}\end{array}$ & $\begin{array}{l}\text { Error } \\
\mathrm{K}\end{array}$ & $\begin{array}{l}\text { Number } \\
\text { of lines }\end{array}$ \\
\hline$\left(\mathrm{CH}_{3}\right)_{2} \mathrm{O}$ & $1.410^{16}$ & $1.810^{15}$ & 157 & 30 & $27(26)$ lines \\
\hline $\mathrm{C}_{2} \mathrm{H}_{3} \mathrm{CN}$ & $3.010^{17}$ & $3.210^{17}$ & 180 & 47 & 13(6) lines \\
\hline $\mathrm{C}_{2} \mathrm{H}_{5} \mathrm{CN}$ & $1.910^{17}$ & $8.410^{16}$ & 103 & 12 & $27(23)$ lines \\
\hline \multirow[t]{2}{*}{$\mathrm{C}_{2} \mathrm{H}_{5} \mathrm{OH}$} & $5.610^{16}$ & $4.010^{16}$ & 70 & - & $8(6)$ lines \\
\hline & $4.110^{16}$ & $2.310^{16}$ & 264 & 196 & $8(6)$ lines \\
\hline $\mathrm{CH}_{2} \mathrm{NH}$ & $2.410^{15}$ & $5.910^{14}$ & 150 & - & $3(2)$ lines \\
\hline $\mathrm{CH}_{3} \mathrm{CN}$ & $3.510^{15}$ & $4.210^{14}$ & 227 & 21 & 19 lines \\
\hline \multirow{2}{*}{$\mathrm{CH}_{3}{ }^{13} \mathrm{CN}$} & $6.010^{14}$ & $2.210^{14}$ & 227 & - & 2 lines \\
\hline & $2.910^{15}$ & - & 74 & - & 2 lines \\
\hline $\mathrm{CH}_{3} \mathrm{OH}$ & $9.310^{16}$ & $4.810^{16}$ & 599 & 295 & $24(23)$ lines \\
\hline $\mathrm{H}_{2} \mathrm{CO}$ & $1.610^{16}$ & - & 166 & - & 2(1) lines \\
\hline $\mathrm{H}_{2}{ }^{13} \mathrm{CO}$ & $1.010^{15}$ & $4.010^{14}$ & 166 & - & 2 lines \\
\hline $\mathrm{HC}_{3} \mathrm{~N}$ & $1.510^{15}$ & - & 164 & - & 2 lines \\
\hline $\mathrm{HCOOCH}_{3}$ & $5.110^{16}$ & $9.510^{15}$ & 301 & 95 & 26(24) lines \\
\hline $\mathrm{HNCO}$ & $4.910^{15}$ & $4.010^{14}$ & 150 & 14 & 4(3) lines \\
\hline \multirow[t]{2}{*}{$\mathrm{NH}_{2} \mathrm{CN}$} & $3.310^{15}$ & $8.510^{14}$ & 200 & - & 3 lines \\
\hline & $1.110^{16}$ & $4.810^{15}$ & 100 & - & 3 lines \\
\hline OCS & $9.010^{16}$ & - & 106 & - & 2 lines \\
\hline SO & $3.310^{17}$ & - & 72 & - & 2(1) lines \\
\hline${ }^{34} \mathrm{SO}$ & $1.110^{16}$ & $3.510^{15}$ & 89 & 43 & 5(4) lines \\
\hline $\mathrm{SO}_{2}$ & $9.710^{16}$ & $1.010^{16}$ & 136 & 9 & $33(28)$ lines \\
\hline $\mathrm{SO}_{2} v=1$ & $7.410^{14}$ & - & 99 & - & 2 lines \\
\hline${ }^{34} \mathrm{SO}_{2}$ & $8.510^{15}$ & - & 156 & - & 3(2) lines \\
\hline${ }^{13} \mathrm{CS}$ & $2.310^{14}$ & - & 120 & - & 1 line \\
\hline${ }^{30} \mathrm{SiO}$ & $3.410^{14}$ & - & 50 & - & 1 line \\
\hline $\mathrm{CH}_{3} \mathrm{CHO}$ & $1.110^{16}$ & - & 81 & - & 1 line \\
\hline $\mathrm{CI}$ & $1.210^{18}$ & - & 30 & - & 1 line \\
\hline $\mathrm{CO}$ & $3.510^{18}$ & - & 200 & - & 4 lines ( 1 transition) \\
\hline $\mathrm{DCN}$ & $1.110^{14}$ & - & 200 & - & 1 line \\
\hline $\mathrm{HCOOH}$ & $2.210^{15}$ & - & 100 & - & 1 line \\
\hline $\mathrm{HDO}$ & $3.210^{16}$ & - & 164 & - & 1 line \\
\hline $\mathrm{N}_{2} \mathrm{O}$ & $4.610^{16}$ & - & 230 & - & 1 line \\
\hline $\mathrm{NH}_{2} \mathrm{CHO}$ & $7.510^{15}$ & - & 81 & - & 1 lines \\
\hline $\mathrm{NH}_{2} \mathrm{D}$ & $8.710^{15}$ & - & 160 & - & 1 line \\
\hline
\end{tabular}



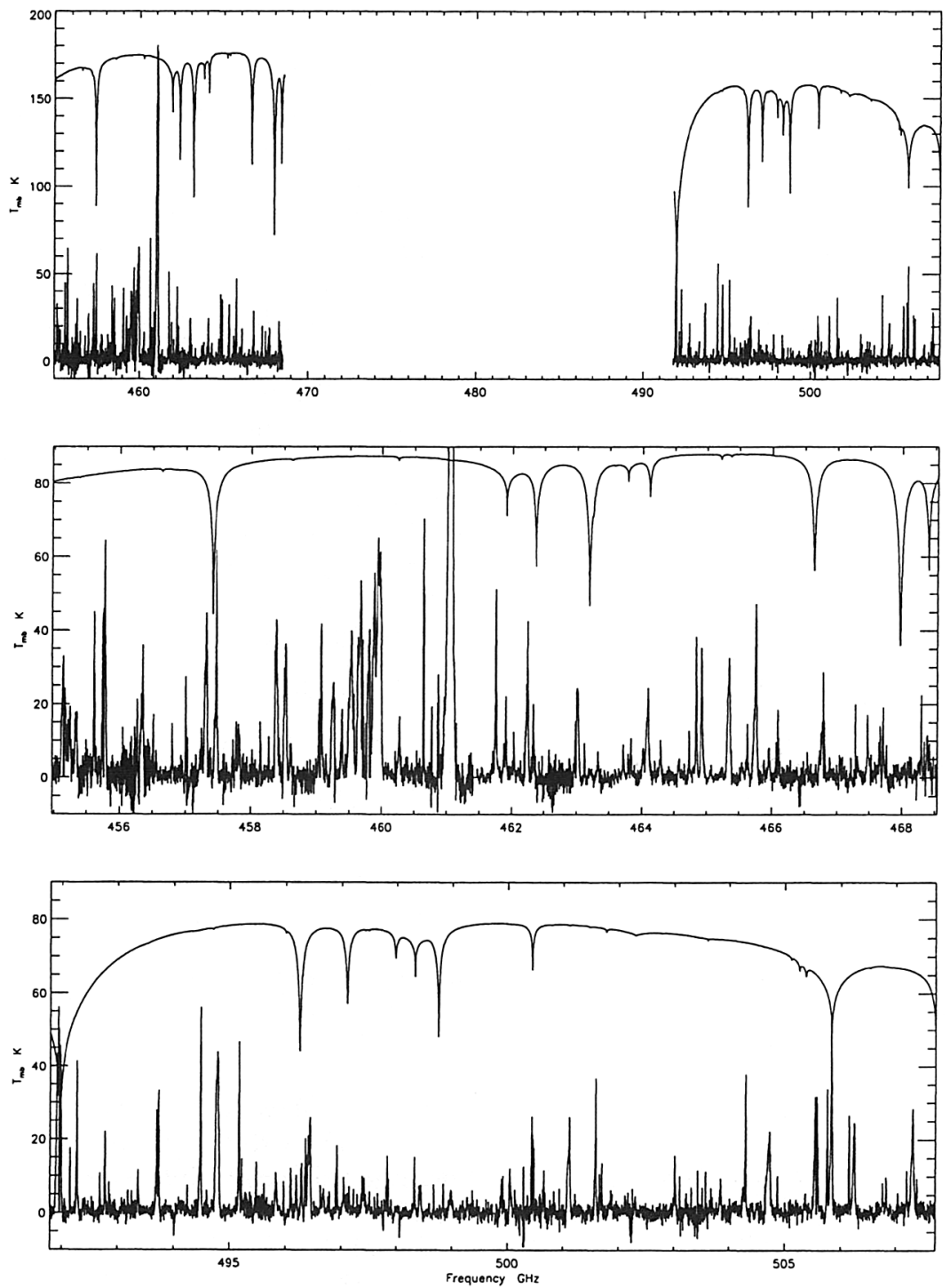

Figure 1. The complete spectrum binned in $2 \mathrm{MHz}$ channels in main beam brightness temperature units. The atmospheric absorption spectrum is overlaid onto the Orion core spectrum. The atmospheric model used to make the absorption spectrum was represented by a 30 layer model, with absorbing material distributed between 4.2 and $80 \mathrm{~km}$ altitude, each having an uniform temperature, density, pressure and molecular concentration - as interpreted from the US Standard Atmosphere model. 


\section{Details of the Survey}

The spectral line survey was made using the James Clerk Maxwell Telescope (JCMT) in Hawaii during October 1993 over the frequency range 455.1 - 507.4 $\mathrm{GHz}$. The spectrum shown in Fig. 1 is crowded with many blended lines, and in many places is confused - reflecting a rich and complex chemistry. A total of 254 lines were identified using the JPL Sub-millimetre spectral line catalogues of Pickett et al. (1995) and Pearson et al. (1996) along with an up to date list of spectral line frequencies available on the JCMT web site. Table 1 gives the breakdown of the number of transitions observed from each species, and the key results are summarised below:

LTE rotation temperatures and beam averaged column density, estimated using a Boltzmann plot. The column densities were determined using the main beam brightness temperature scale, and for species where only one line was measured, we have assumed a rotational temperature as described in Column 7. Notes: Column 6: A(B) lines represent A: number of assigned lines, B: number of lines included in the fitting. For A lines only, all lines were included in the fitting [1] : $v_{2}=1$ state lines were not included in the fitting, [2] : Energy levels were considered in the $v=1$ vibrational state. Errors quoted are all $1 \sigma$.

- A total of 254 lines were detected towards the hot core position at the centre of the Orion Nebula.

- Spectral lines of $\mathrm{SO}, \mathrm{SO}_{2}$ and $\mathrm{CH}_{3} \mathrm{OH}$ and from large organic molecules such as $\left(\mathrm{CH}_{3}\right)_{2} \mathrm{O}, \mathrm{CH}_{3} \mathrm{CN}, \mathrm{C}_{2} \mathrm{H}_{5} \mathrm{CN}, \mathrm{HCOOCH}{ }_{3}$ and $\mathrm{C}_{2} \mathrm{H}_{3} \mathrm{CN}$ make up $\sim 72 \%$ of the total number of lines

- A total of 33 lines ( $13 \%$ of the total lines identified) could not be associated with known molecular transitions and are designated U-lines.

- The rotational temperatures and column densities were derived using standard rotation diagram analysis techniques, and found to range from $70-300 \mathrm{~K}$, and $10^{14}-10^{17} \mathrm{~cm}^{2}$ respectively.

The detection of compounds such as aldehydes, ketones, hydrocarbons, and polycyclic aromatic hydrocarbons in the interstellar medium shows that the material that could have contributed to the organic chemistry that led to life on the Earth is also present in other regions of space where stars are being formed. Although this does not lead to definite conclusions about the existence of life elsewhere, it does hint strongly at the likelihood of the possibility of life forming elsewhere.

\section{References}

Pearson, J. C., Sastry, K. V. L. N., Herbst, E., \& De Lucia, F.J. 1996, J. Mol. Spect., 175, 246

Pickett, H. M., Poyner R. L., \& Cohen E. A. 1995, in Submillimeter, Millimeter, and Microwave Spectral line Catalogue, http://spec.jpl.nasa.gov 